\title{
Limits on the limitations of context-conditioned effects in the perception of [b] and [w]
}

\author{
JOANNE L. MILLER and SARAH C. WAYLAND \\ Northeastern University, Boston, Massachusetts
}

\begin{abstract}
We investigated the conditions under which the $[b]-[w]$ contrast is processed in a contextdependent manner, specifically in relation to syllable duration. In an earlier paper, Miller and Liberman (1979) demonstrated that when listeners use transition duration to differentiate [b] from [w], they treat it in relation to the duration of the syllable: As syllables from a [ba]-[wa] series varying in transition duration become longer, so, too, does the transition duration at the [b]-[w] perceptual boundary. In a subsequent paper, Shinn, Blumstein, and Jongman (1985) questioned the generality of this finding by showing that the effect of syllable duration is eliminated for [ba]-[wa] stimuli that are less schematic than those used by Miller and Liberman. In the present investigation, we demonstrated that when these "more natural" stimuli are presented in a multitalker babble noise instead of in quiet (as was done by Shinn et al.), the syllable-duration effect emerges. Our findings suggest that the syllable-duration effect in particular, and context effects in general, may play a more important role in speech perception than Shinn et al. suggested.
\end{abstract}

There is widespread evidence for context-dependent processing in speech perception. One context effect, and the focus of the current paper, is the syllable-duration effect reported by Miller and Liberman (1979) for the distinction between syllable-initial stop consonant [b] and semivowel [w].

It has been known for some time that a major acoustic property distinguishing [b] and [w] is the duration of the initial formant transitions and that this property is functional in perception. All else being equal, listeners identify stimuli with short transitions as [b] and those with longer transitions as [w] (see, e.g., Liberman, Delattre, Gerstman, \& Cooper, 1956). However, a change in context can alter the precise mapping between transition duration and the $[b]-[w]$ contrast. In particular, a talker's rate of speech influences the specific ranges of transition durations produced for the two phonetic segments. As speakers slow down, such that the individual syllables become longer, the initial formant transitions (especially those for [w]) also become longer (Miller \& Baer, 1983). Moreover, Miller and Liberman (1979) have shown that listeners are sensitive to this contextual variation. This is seen as a shift in the perceptual boundary between [b] and $[w]$ toward longer transition durations, as syllables become longer. Miller and Liberman interpreted this perceptual context effect in terms of a normalization for

This research was supported by NIH Grant DC 00130 to J. L. Miller, NIH Grant DC 00043 to S. C. Wayland, and NIH Biomedical Research Support Grant RR 07143. We thank P. C. Shinn, S. E. Blumstein, and S. A. Jongman for providing us with their stimuli, Peter D. Eimas for valuable comments on an earlier version of the manuscript, and Lauren Forget for assistance in data collection. Correspondence should be addressed to Joanne L. Miller, Department of Psychology, Northeastern University, Boston, MA 02115 (e-mail: jlmiller@nuhub.bitnet). speaking rate: The duration of the syllable specifies speaking rate, and listeners use this rate information when making the phonetic judgment of $[b]$ versus $[w] .{ }^{1}$

The syllable-duration effect for $[b]$ versus $[w]$ has been replicated many times (see, e.g., Diehl \& Walsh, 1989; Pisoni, Carrell, \& Gans, 1983); it has been found for young, prearticulate infants (Eimas \& Miller, 1980; Miller \& Eimas, 1983); and comparable effects have been found for other phonetic contrasts (see, e.g., Miller \& Volaitis, 1989; Summerfield, 1981). It should be noted, however, that although the syllable-duration effect has proved robust, the original interpretation offered by Miller and Liberman (1979) has been questioned. For example, it has been proposed that although the syllable-duration effect may serve the important function of accommodating for changes in speaking rate, it does not derive from a normalization for speaking rate, per se, but rather is due to the operation of general auditory processes that apply to speech and nonspeech signals alike (Diehl \& Walsh, 1989; Jusczyk, Pisoni, Reed, Fernald, \& Myers, 1983; Pisoni et al., 1983; cf. Stevens, Kuhl, \& Padden, 1988). However, this interpretation has itself been called into question (Fowler, 1990; but see Diehl, Walsh, \& Kluender, 1991), and at the present time the underlying basis of the effect remains controversial.

The present investigation focuses on a different issue regarding the syllable-duration effect, namely, the conditions under which it occurs. Our research derives from a paper by Shinn, Blumstein, and Jongman (1985), who proposed that the role of context effects in speech perception, including the syllable-duration effect, might be overestimated because of the schematic nature of the stimuli typically used to assess such effects. They noted that the stimuli used in the Miller and Liberman (1979) paper (as well as those used in the follow-up studies cited 
above) were rather stylized versions of [ba] and [wa]. In particular, the change from $[b]$ to $[w]$ was specified by a change in duration of the initial formant transitions, with concomitant changes in the rate of the formant transitions and the abruptness of the amplitude onset, all factors that covary in the natural production of [b] versus [w]. However, other acoustic properties that differentiate [b] from $[w]$ in natural speech, such as the onset frequencies of the formant transitions, were not varied. Shinn et al. (1985) hypothesized that the syllable-duration effect might disappear if the stimuli varied in these other acoustic properties, which presumably are less influenced by contextual variables (such as changes in speaking rate) than is transition duration. That is, listeners might perceive speech in a context-dependent manner-thus showing the syllable-duration effect-if only properties that acoustically vary with context are available in the stimuli to specify the $[b]-[w]$ distinction, but will perceive the stimuli in a context-independent manner-showing no syllable-duration effect-if properties that do not vary with context are available for making the $[b]-[w]$ distinction.

To test this hypothesis, Shinn et al. (1985) asked listeners to make $[b]-[w]$ identification judgments for four sets of [ba]-[wa] series. Each set contained five [ba]-[wa] series that differed from one another in overall syllable duration. The sets differed from each other in the way in which the $[b]-[w]$ contrast was specified. One set was closely patterned after the Miller and Liberman (1979) stimuli, with the $[b]-[w]$ contrast specified by transition duration, with concomitant variation in transition rate and abruptness of amplitude onset. Across the other three sets, additional parameters were varied until by the fourth set the stimuli were closely patterned after natural speech, with numerous properties, including the onset frequencies of the formant transitions and the detailed changes in formant trajectories, differentiating [b] and [w]. The main finding was that the syllable-duration effect was replicated for the stimuli patterned after those used by Miller and Liberman, but became smaller as the stimuli became less schematic, with no effect for the stimuli most closely patterned after natural speech. Shinn et al. (1985) took this finding as support for the view that context effects such as the syllable-duration effect may play only a limited role in the perception of natural speech.

In the current experiment, we investigated the possibility that the role of the syllable-duration effect is not as limited as Shinn et al. (1985) suggested. Specifically, we examined whether there are conditions under which the effect emerges even for stimuli closely patterned after natural speech. This prospect is based on the finding that a change in listening conditions can alter the functional significance, or importance, of each of the multiple properties that specify a given phonetic contrast. Consider as an example the voicing distinction in syllable-final position, as in [bId] versus [bIt]. One of the properties specifying this contrast is preceding vowel duration, which is longer for voiced than voiceless consonants. WardripFruin (1985) has shown that the importance of this acoustic property varies with changes in listening conditions.
Specifically, the importance of preceding vowel duration increases considerably when the stimuli to be identified are presented in the context of a multitalker babble noise instead of in quiet. In the current experiment, we asked whether an analogous situation exists for the contribution of transition duration to the specification of the $[b]-[w]$ contrast.

The idea is this: There is evidence that the listener's adjustment for changes in speaking rate is obligatory (Miller, 1987; Miller \& Dexter, 1988; Miller, Green, \& Schermer, 1984). Assume, then, that whenever listeners use transition duration to specify the $[b]-[w]$ contrast, they treat this property in a rate-dependent manner, specifically, in relation to the overall duration of the syllable. This yields the syllable-duration effect. However, when other properties are available to make the distinction-as in the Shinn et al. (1985) stimuli patterned after natural speech-transition duration plays a relatively minor role, and thus no syllable-duration effect is seen. A syllableduration effect is seen for the stimuli used by Miller and Liberman (1979) because other potential properties have been neutralized. The interesting question is what would occur if the two sets of stimuli were presented in the context of a multitalker babble noise, such as that used by Wardrip-Fruin (1985). The syllable-duration effect should be maintained for the Miller and Liberman stimuli, because transition duration is still the major property distinguishing the contrast. The critical case concerns the Shinn et al. stimuli patterned after natural speech. If transition duration behaves like preceding vowel duration, then it will be more important in the presence of noise than in quiet. Given the assumption that transition duration is always processed in relation to overall syllable duration, the syllable-duration effect should emerge.

We tested this hypothesis by assessing perception of two sets of stimuli from Shinn et al. (1985), the set patterned after Miller and Liberman (1979) and the set patterned most closely after natural speech. We assessed identification of these stimuli in quiet, which is a replication of Shinn et al., and also in the presence of a multitalker babble noise. We expected to obtain the syllable-duration effect for the Miller and Liberman set in both quiet and noise. We also predicted that even though the Shinn et al. stimuli would not show the effect in quiet (thus replicating Shinn et al., 1985), they would show the effect in noise.

\section{METHOD}

\section{Subjects}

Eighty students and staff from the Northeastern University community served as paid subjects in the experiment, 20 in each of the four experimental conditions. All subjects passed an audiometric screening test.

\section{Stimuli}

Two sets of syllables from Shinn et al. (1985), kindly provided to us by the authors, were used in the experiment. We obtained the stimuli on audiotape and subsequently digitized them $(10-\mathrm{kHz}$ sampling rate, $4.8-\mathrm{kHz}$ lowpass filtering) for on-line presentation to subjects. 
One set of stimuli was from Condition 1 (Series 1) in Shinn et al (1985). These stimuli were patterned after those originally used by Miller and Liberman (1979) and will be referred to as the ML set. The other set was from Condition 4 (Series 1) in Shinn et al. These were less stylized than the ML stimuli and were patterned more closely after natural speech. These will be referred to as the SBJ set. In Shinn et al., the ML and SBJ sets were each composed of five [ba]-[wa] series that differed from each other in syllable duration. The durations ranged from 102 to $317 \mathrm{msec}$ for the ML set and from 85 to $445 \mathrm{msec}$ for the SBJ set. For the present experiment, we selected two series from each set for testing. These were the 102-and 248-msec stimuli from the ML set, which we will refer to as the short and long ML stimuli, respectively, and the 85- and 265-msec stimuli from the SBJ set, which we will refer to as the short and long SBJ stimuli, respectively. With these stimuli, there is a substantial difference in syllable duration within each set, whereas syllable durations are reasonably similar across sets (with the absolute difference in syllable duration being somewhat larger for the SBJ set than for the ML set).

Detailed acoustic specifications of the ML and SBJ stimuli can be found in Shinn et al. (1985). Briefly, the stimuli can be characterized as follows. They were all five-formant patterns generated with the cascade version of the Klatt (1980) software synthesizer. For the ML set, the major acoustic property specifying the change from [b] to [w] across the 11-member series was the duration of the initial formant transitions, with a correlated change in transition rate and abruptness of amplitude onset; the transitions (and amplitude onset) became longer and more gradual across the series. ${ }^{2}$ For the SBJ set, the acoustic modification across the 11-member series specifying the change from [b] to [w] was considerably more complex. Most notably, in addition to a change in the duration and rate of the initial formant transitions and a change in the amplitude characteristics at stimulus onset, there was also a change in the onset frequency values of the formants, resulting in a complex change in formant trajectories. Across the series the transitions (and amplitude onset) became more gradual, and the formant-transition onset values became lower. In all conditions of the current experiment, the ML and SBJ syllables were presented to the subjects binaurally over Yamaha YH-1 earphones at $77 \mathrm{~dB}$ SPL.

The multitalker babble was a slightly modified version of the 12talker babble developed for the SPIN test (Kalikow, Stevens, \& Elliott, 1977). The babble was presented from audiotape and mixed with the analogue speech signal prior to earphone input. The babble was presented at $63 \mathrm{~dB}$ SPL, to yield a signal-to-noise ratio of $14 \mathrm{~dB}$.

\section{Procedure}

The experiment consisted of four conditions resulting from the factorial combination of two factors, a noise manipulation (quiet vs. noise) and a manipulation of presentation format (separate vs. mixed). In the two noise conditions, the ML and SBJ syllables were presented in the presence of a 12-talker babble noise at a signal-tonoise ratio of $14 \mathrm{~dB}$, as described above. In the two quiet conditions, they were presented without the babble noise. In the two separate conditions, the ML and SBJ stimuli were tested in separate blocks. This allows for a replication of the Shinn et al. (1985) study in quiet (in which the ML and SBJ stimuli were presented separately) and a straightforward comparison with presentation in noise. In the two mixed conditions, the SBJ and ML stimuli were intermixed and presented together. This condition was included to test the possibility (although unlikely) that the simple presence of the ML stimuli in the test sequence would increase the importance of transition duration in the SBJ stimuli, yielding the syllable-duration effect. Finally, note that in all four conditions, the short and long stimuli were randomly mixed together for presentation; this corresponds to the mixed format of Shinn et al.

A different group of 20 subjects participated in each of the four conditions, quiet/separate, quiet/mixed, noise/separate, and noise/mixed. Each subject was tested separately in a session that lasted approximately $1 \mathrm{~h}$. For the two separate conditions, the session was divided into two parts, one part devoted to the ML stimuli and the other to the SBJ stimuli. Each part included practice and test trials. For the practice trials, the subjects identified one randomized block of the 22 stimuli ( 11 short stimuli and 11 long stimuli). The primary purpose of the practice was to familiarize the subjects with the stimuli and procedure; no feedback was provided. For the test trials, the subjects identified 15 randomized blocks of the 22 stimuli, for a total of 330 trials. A short break was given in the middle of the test run. Half of the subjects identified the ML stimuli first and the SBJ stimuli second, and the other half identified the stimuli in the reverse order. For the two mixed conditions, the session consisted of one set of practice and one set of test trials. The practice trials included one randomized block of the 44 stimuli (22 ML stimuli, 11 short and 11 long, and 22 SBJ stimuli, 11 short and 11 long). The test trials included 15 randomized blocks of the 44 stimuli, for a total of 660 trials. Two short breaks were given during the test run. For all conditions, the subjects indicated their response, [ba] or [wa], by pressing the appropriately labeled key on a computer keyboard. The intertrial interval was $2 \mathrm{sec}$, timed from the subject's keypress to the onset of the next syllable.

\section{RESULTS}

The group data are displayed in Figures 1 and 2 for the ML and SBJ stimuli, respectively. Within each figure, four sets of identification functions are presented, corresponding to the four experimental conditions. The main findings are clear. There was an effect of syllable duration for the ML stimuli in all conditions. In contrast, syllable duration did not affect perception of the SBJ stimuli when presented in quiet; however, when these stimuli were presented in noise, the effect emerged.

The statistical analyses supporting these conclusions are as follows. First, for each subject in each condition, we computed the location of the [b]-[w] category boundary (in terms of stimulus number, 1-11) for the short and long ML and SBJ stimuli, yielding four boundary values per

\section{STIMULI}
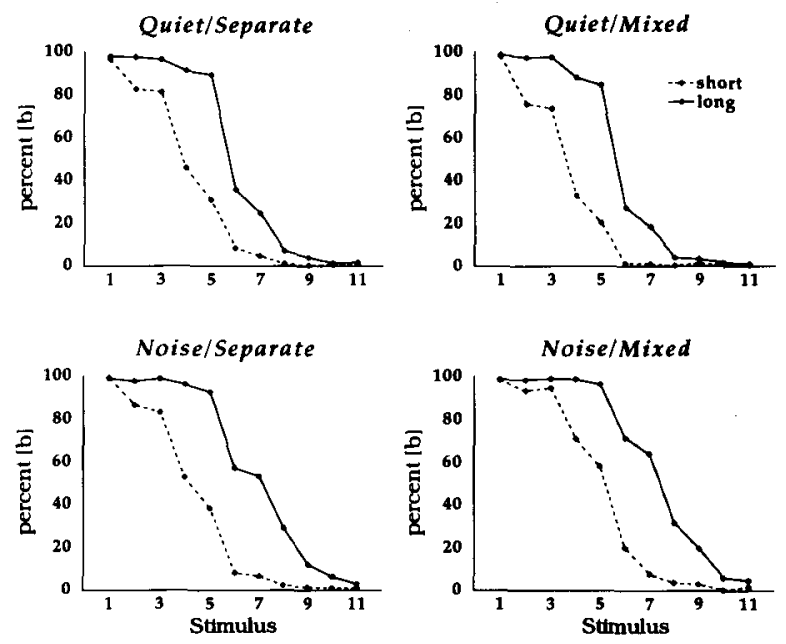

Figure 1. Group identification functions for the short and long ML syllables in four experimental conditions. 


\section{SBJ STIMULI}
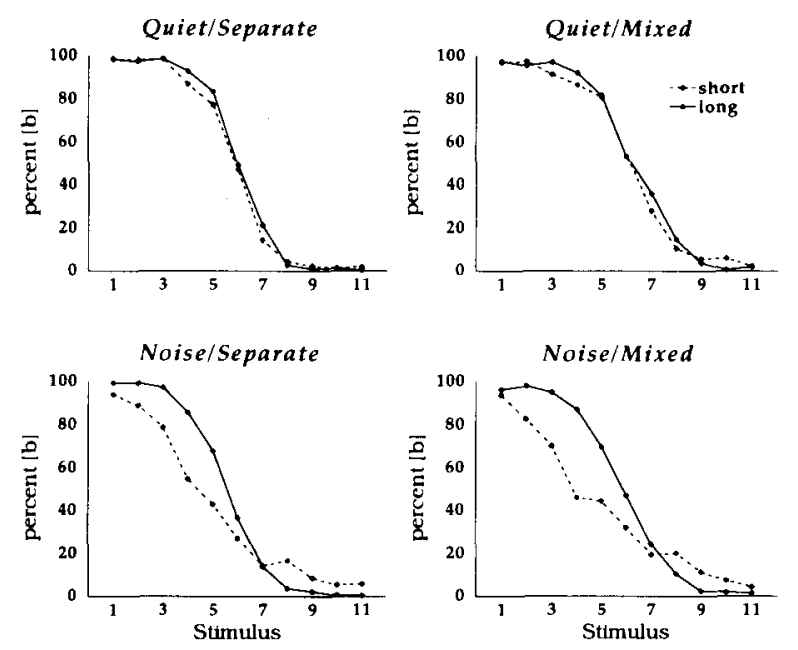

Figure 2. Group identification functions for the short and long SBJ syllables in four experimental conditions.

subject. The boundary values were determined by fitting a linear regression line to the data in the boundary region of the function (defined as the region between $90 \%$ and $10 \%$ [ba] responses) and taking the boundary to be the stimulus value equivalent to $50 \%$ responses. The group means for these boundary values are given in Table 1 . Second, two separate analyses of variance (ANOVAs) were carried out on the individual boundary values, one for the ML stimuli and one for the SBJ stimuli. ${ }^{3}$ For each ANOVA, noise level (noise vs. quiet) and presentation format (mixed vs. separate) were between-subject variables and syllable duration (short vs. long) was a withinsubject variable.

Consider first the ML stimuli. There was a highly reliable effect of syllable duration $[F(1,76)=709, p<$ $.001]^{4}$ with the boundary located at a higher stimulus value for the long compared with the short stimuli. The size of this effect varied somewhat across conditions, as indicated by a reliable syllable duration $x$ noise interaction $[F(1,76)=7.99, p<.01]$, which was due to a larger syllable-duration effect in the presence of noise than in

Table 1

Mean Boundary Values (in Stimulus Number) for the Short and Long ML and SBJ Stimuli, and Mean Difference Scores, in Each of Four Experimental Conditions

\begin{tabular}{|c|c|c|c|c|}
\hline & \multicolumn{2}{|c|}{ Quiet } & \multicolumn{2}{|c|}{ Noise } \\
\hline & Separate & Mixed & Separate & Mixed \\
\hline $\begin{array}{l}\text { ML_Short } \\
\text { ML_Long }\end{array}$ & $\begin{array}{l}4.03 \\
5.98\end{array}$ & $\begin{array}{l}3.54 \\
5.74\end{array}$ & $\begin{array}{l}4.23 \\
6.98\end{array}$ & $\begin{array}{l}4.99 \\
7.38\end{array}$ \\
\hline Difference & $1.95 *$ & $2.20^{*}$ & $2.75^{*}$ & $2.39^{*}$ \\
\hline $\begin{array}{l}\text { SBJ_Short } \\
\text { SBJ-Long }\end{array}$ & $\begin{array}{l}5.77 \\
5.95\end{array}$ & $\begin{array}{l}6.07 \\
6.29\end{array}$ & $\begin{array}{l}4.83 \\
5.56\end{array}$ & $\begin{array}{l}4.68 \\
5.82\end{array}$ \\
\hline Difference & $0.18 \ddagger$ & $0.22 \ddagger$ & $0.73 t$ & $1.14^{*}$ \\
\hline
\end{tabular}

${ }^{*} p<.01 . \quad+p<.05 . \quad \neq p>.10$. quiet. Most importantly, individual comparisons (Tukey $H S D$ ) revealed that in all four experimental conditions, the syllable-duration effect was highly significant ( $p s<$ $.01)$. Finally, there was also a reliable effect of noise $[F(1,76)=38.65, p<.001]$, as well as a presentation format $\times$ noise interaction $[F(1,76)=7.47, p<.01]$; these two effects were due to the boundary being shifted toward higher stimulus values in the presence of noise, but only with the mixed presentation format. The main effect of presentation format $(p>.10)$, the interaction of presentation format with syllable duration $(p>.10)$, and the three-way interaction of syllable duration, noise, and presentation format $(p>.08)$ were all nonsignificant.

Next turn to the SBJ stimuli. For these stimuli, there was a reliable syllable-duration effect $[F(1,76)=26.16$, $p<.001]$ and a reliable noise effect $[F(1,76)=14.63$, $p<.001]$, which was due to the boundary being shifted toward lower stimulus values in the presence of noise (for both the short and long stimuli). ${ }^{5}$ Importantly, there was also a reliable syllable duration $\times$ noise interaction $[F(1,76)=10.95, p<.005]$. Individual comparisons (Tukey $H S D$ ) indicated that this was due to the fact that the effect of syllable duration was not significant in either the quiet/separate or quiet/mixed condition $(p s>.10)$ but was significant in both the noise/separate $(p<.05)$ and the noise/mixed $(p<.01)$ conditions. Presentation format and all interactions with presentation format were not significant $(p s>.10)$.

The main results can be summarized as follows. For the ML stimuli, there was a reliable effect of syllable duration in all four conditions. For the SBJ stimuli, there was no reliable effect in the two quiet conditions, but there was a reliable effect in the two noise conditions. For neither set of stimuli did presentation format affect the size or presence of the syllable-duration effect.

\section{DISCUSSION}

Our results underscore the role of context effects in speech perception. We have shown that a particular form of context dependency, the syllable-duration effect reported by Miller and Liberman (1979) for the $[b]-[w]$ contrast, does not depend on the stimuli being schematic in nature, as suggested by Shinn et al. (1985). Rather, the effect is obtained even for more complex stimuli patterned closely after natural speech, if the stimuli are presented not in quiet but instead in a multitalker babble noise. Not surprisingly, we also found that the syllable-duration effect is obtained for schematic stimuli such as those used by Miller and Liberman in both noise and quiet.

The critical question is why the context effect occurs for the more complex stimuli (the SBJ stimuli) only in noise, but is seen for the schematic stimuli (the ML stimuli) regardless of listening conditions. We suggest that the reason has to do with the status of transition duration in specifying the contrast in the two sets of stimuli, in the quiet versus noise conditions. Consider first the quiet conditions. In the ML stimuli, transition duration is the major property specifying the distinction. Formant fre- 
quency onset, which covaries with [b] and [w] in natural speech, is constant. Given the assumption that transition duration is obligatorily processed in relation to syllable duration (cf. Miller, 1987; Miller \& Dexter, 1988), then, because the $[b]-[w]$ decision is based primarily on transition duration, a syllable-duration effect is seen. The situation for the SBJ stimuli is quite different. In these stimuli, the $[b]-[w]$ contrast is specified not only by transition duration, but also by a host of properties differentiating [b] from [w] in natural speech, including formant frequency onset-properties that, unlike transition duration, are presumably processed without reference to syllable duration. We propose that given the availability of these other properties, transition duration, per se, played only a minor role in $[b]-[w]$ categorization. Consequently, the effect of syllable duration was not seen.

Consider, however, what happens when the stimuli are presented in noise. As we outlined in the introduction, a change in listening conditions, specifically, a change from quiet to babble noise, can alter the relative importance of acoustic properties specifying a given phonetic contrast. In particular, Wardrip-Fruin (1985) has shown that preceding vowel duration becomes a particularly important cue to the postvocalic voicing distinction when the stimuli are presented in noise. We propose that an analogous situation occurred for the SBJ stimuli: When they were presented in noise, transition duration became more important. And because transition duration is obligatorily processed in relation to syllable duration, the syllable-duration effect emerged. Interestingly, noise may have played a similar role in the perception of the ML stimuli. Although there was a reliable syllable-duration effect in both quiet and noise for these stimuli, the effect was larger in noise. Perhaps this was also due to a change in the importance of transition duration in specifying the contrast, but in this case as regards amplitude onset. As we have noted, for the ML stimuli (as for the SBJ stimuli), transition duration covaried with the abruptness of the amplitude onset. Although somewhat controversial, it appears that amplitude onset is at best a relatively weak cue for the [b]-[w] contrast (Diehl \& Walsh, 1989; Nittrouer \& Studdert-Kennedy, 1986; Walsh \& Diehl, 1991; but see Shinn \& Blumstein, 1984). Moreover, it apparently is not processed in relation to syllable duration (Diehl $\&$ Walsh, 1989). Perhaps, then, in the quiet conditions, amplitude onset did play a minor role in specifying the contrast for the ML stimuli, but in the noise conditions, its role was diminished even further, such that transition duration became more important. Because the syllableduration effect is mediated by transition duration, it became larger. ${ }^{6}$

On the above account, the effect of babble noise is to change the importance of transition duration as a cue to the $[b]-[w]$ contrast. The reason for this change is not known, but we can offer two possibilities. One concerns the masking properties of the babble noise. Perhaps the temporal property of transition duration is particularly robust to the effects of masking compared with the other properties cuing the distinction, such as the spectral property of formant onset frequency (cf. Wardrip-Fruin, 1985). As a consequence, in the presence of noise, these other properties lose effectiveness, and the importance of transition duration increases. Alternatively, perhaps the change is due not to the noise, per se, but to the increased difficulty of listening to speech in noise. Gordon, Eberhardt, and Rueckl (1993) have recently shown that altering the difficulty of the listening situation by introducing a concurrent distractor task can change the relative importance of various properties underlying a phonetic distinction. Perhaps in the current study the babble noise induced a similar change, with transition duration becoming more important. ${ }^{7}$

In conclusion, our findings show that the syllableduration effect obtains even for stimuli closely patterned after natural speech, if the stimuli are presented in the context of a multitalker babble noise. Given that such background noise is arguably more similar to natural listening conditions than is a quiet setting, these findings suggest that context effects do play an important role in speech perception. The challenge before us is to explicate the nature of the perceptual mechanisms that underlie these effects.

\section{REFERENCES}

DieHL, R. L., \& WALSH, M. A. (1989). An auditory basis for the stimulus-length effect in the perception of stops and glides. Journal of the Acoustical Society of America, 85, 2154-2164.

DieHL, R. L., WALSH, M. A., \& KLUENDER, K. R. (1991). On the interpretability of speech/nonspeech comparisons: A reply to Fowler Joumal of the Acoustical Society of America, 89, 2905-2909.

EIMAS, P. D., \& MiLler, J. L. (1980). Contextual effects in infant speech perception. Science, 209, 1140-1141.

FowlER, C. A. (1990). Sound-producing sources as objects of perception: Rate normalization and nonspeech perception. Journal of the Acoustical Society of America, 88, 1236-1249.

Gordon, P. C., Eberhardt, J. L., \& Rueckl, J. G. (1993). Attentional modulation of the phonetic significance of acoustic cues. Cognitive Psychology, 25, 1-42.

Jusczyk, P. W., Pisoni, D. B., Reed, M. A., Fernald, A., \& MYERS, M. (1983). Infants' discrimination of the duration of a rapid spectrum change in nonspeech signals. Science, 222, 175-177.

Kalikow, D. N., Stevens, K. N., \& Eluott, L. L. (1977). Development of a test of speech intelligibility in noise using sentence materials with controlled word predictability. Journal of the Acoustical Society of America, 61, 1337-1351.

KLATT, D. H. (1980). Software for a cascade/parallel formant synthesizer. Journal of the Acoustical Society of America, 67, 971-995.

Liberman, A. M., Delattre, P. C., Gerstman, L. J., Cooper, F. S. (1956). Tempo of frequency change as a cue for distinguishing classes of speech sounds. Journal of Experimental Psychology, 52, 127-137.

Miller, J. L. (1987). Mandatory processing in speech perception: A case study. In J. L. Garfield (Ed.), Modularity in knowledge representation and natural-language understanding (pp. 309-322). Cambridge: MIT Press/Bradford Books

Miller, J. L., \& BaER, T. (1983). Some effects of speaking rate on the production of $/ \mathrm{b} /$ and $/ \mathrm{w} /$. Journal of the Acoustical Society of America, 73, 1751-1755.

Miller, J. L., \& Dexter, E. R. (1988). Effects of speaking rate and lexical status on phonetic perception. Journal of Experimental Psychology: Human Perception \& Performance, 14, 369-378. 
Miller, J. L., \& Eimas, P. D. (1983). Studies on the categorization of speech by infants. Cognition, 13, 135-165.

Miller, J. L., Green, K., \& Schermer, T. M. (1984). A distinction between the effects of sentential speaking rate and semantic congruity on word identification. Perception \& Psychophysics, 36, 329-337.

Miller, J. L., \& Liberman, A. M. (1979). Some effects of lateroccurring information on the perception of stop consonant and semivowel. Perception \& Psychophysics, 25, 457-465.

Miller, J. L., \& Volaitis, L. E. (1989). Effect of speaking rate on the perceptual structure of a phonetic category. Perception \& Psychophysics, 46, 505-512.

Nittrouer, S., \& Studdert-Kennedy, M. (1986). The stop-glide distinction: Acoustic analysis and perceptual effect of variation in syllable amplitude envelope for initial $/ \mathrm{b} /$ and $/ \mathrm{w} /$. Joumal of the Acoustical Society of America, 80, 1026-1029.

Pisoni, D. B., Carrell, T. D., \& Gans, S. J. (1983). Perception of the duration of rapid spectrum changes in speech and nonspeech signals. Perception \& Psychophysics, 34, 314-322.

Schwab, E. C., Sawusch, J. R., \& Nusbaum, H. C. (1981). The role of second formant transitions in the stop-semivowel distinction. Perception \& Psychophysics, 29, 121-128.

ShinN, P., \& Blumstein, S. E. (1984). On the role of the amplitude envelope for the perception of [b] and [w]. Journal of the Acoustical Society of America, 75, 1243-1252.

Shinn, P. C., Blumstein, S. E., \& Jongman, A. (1985). Limitations of context conditioned effects in the perception of $[b]$ and $[w] . P e r-$ ception \& Psychophysics, 38, 397-407.

Stevens, E. B., Kuhl, P. K., \& Padden, D. M. (1988). Macaques show context effects in speech perception. Joumal of the Acoustical Society of America, 84, (Suppl. 1), S77-S78.

SUMmeRFIELD, Q. (1981). Articulatory rate and perceptual constancy in phonetic perception. Journal of Experimental Psychology: Human Perception \& Performance, 7, 1074-1095.

WALSH, M. A., \& DiEHL, R. L. (1991). Formant transition duration and amplitude rise time as cues to the stop/glide distinction. Quarterly Journal of Experimental Psychology, 43A, 603-620.

W ARDRIP-Fruin, C. (1985). The effect of signal degradation on the status of cues to voicing in utterance-final stop consonants. Journal of the Acoustical Socien of America, 77, 1907-1912.

\section{NOTES}

1. It should be noted that the effect is actually more complicated. Miller and Liberman (1979) provided evidence that it is not syllable duration, per se, that provides the critical rate information, but rather syllable duration in conjunction with syllable structure (cf. Summerfield, 1981; see also Pisoni et al., 1983). Given that only simple consonant-vowel syllables are used in the current experiment, this issue will not be considered further.

2. In the original Miller and Liberman (1979) stimuli, the decrease in abruptness of amplitude onset with increasing transition duration was accomplished through a change in the synthesis parameters. In contrast, for the ML stimuli, the change in amplitude onset derived from the operation of the synthesizer itself, with amplitude synthesis parameters held constant across the stimuli within the series.

3. Because the acoustic dimensions and step sizes along the series (stimulus number 1-11) are not commensurate for the ML and SBJ stimuli, it was not appropriate to conduct one overall ANOVA with stimulus type (ML vs. SBJ) as a factor. Thus, even though a given subject heard both the ML and SBJ stimuli, these two stimulus types were considered separately in the analyses.

4. All $p$ values from the ANOVAs remain the same with the GeisserGreenhouse correction for sphericity.

5. Note that the presence of noise had the overall effect of shifting the category boundary toward higher values in the ML stimuli, but toward lower values in the SBJ stimuli (see Table 1). The reason for this difference is unknown.

6. As noted earlier, for both the ML and SBJ stimuli, the change in transition duration was accompanied by a change in transition rate. However, there is evidence that transition rate, per se, is not effective in specifying the $[b]-[w]$ contrast (Liberman et al., 1956; Schwab, Sawusch, \& Nusbaum, 1981).

7. We should note, however, that the reason transition duration, per se, would become more important in the difficult listening situation is not clear. Gordon et al. (1993) proposed that under conditions of reduced attention, "weak" cues to phonetic contrasts become more important and "strong" cues become less important, but there is no obvious basis for classifying transition duration as a "weak" cue.

(Manuscript received May 26, 1992;

revision accepted for publication January 27, 1993.) 